\title{
Malformaciones uterinas y fertilización in -vitro: resultados después de histeroplastia histeroscópica
}

\author{
Christian Manuel Pérez-Villabona ${ }^{1}$; Hermes Jaimes Carvajal ${ }^{2}$; Miguel Angel Alarcón Nivia ${ }^{3}$; \\ Claudia Patricia Parra Barón ${ }^{4}$; Harold Maldonado Cárdenas ${ }^{5}$; Bernard Blanc ${ }^{6}$; Leon Boubli ${ }^{7}$
}

\section{RESUMEN}

OBJETIVOS DEL ESTUDIO: Determinar la efectividad del tratamiento quirúrgico endoscópico en pacientes con malformaciones uterinas sometidas a fertilización in-vitro, medida en tasa de embarazos.

DISEÑO: Es un estudio de cohortes retrospectivo en un período de siete años, desde 1990 a 1997.

MATERIALES Y METODOS: Para efectos del estudio se tomaron 16 pacientes con tabiques uterinos, de los cuales 9 recibieron tratamiento quirúrgico endoscópico y 7 permanecieron sin tratamiento. Se utilizaron los test de Chi Cuadrado, Chi Cuadrado con corrección de Yates y test de Fisher teniendo en cuenta el tamaño de la muestra.

RESULTADOS: La edad media de las pacientes fue de 33,4 años. La tasa de embarazo fue de $44.4 \%$ en el grupo con tratamiento endoscópico quirúrgico contra un $\mathbf{4 2 . 8 \%}$ en el grupo no tratado, observándose que la tasa de embarazos fue similar en ambos grupos sin diferencia estadísticamente significativa ( $>\mathbf{0 . 0 5}$ ). Pero se observó que la tasa de embarazos evolutivos normales que llegaron a termino fue significativamente mayor en el grupo tratado quirúrgicamente que en el no tratado, $44.4 \%$ vs $14.2 \%$.

CONCLUSIONES: Los resultados observados nos conducen a concluir que existe una indicación para el tratamiento quirúrgico endoscópico de los tabiques uterinos antes de planear un intento de fertilización in-vitro, ya que este mejora la tasa de embarazo evolutivo normal que llega a termino; al parecer por una mejoría en la capacidad y adecuación de la cavidad uterina. Así mismo, teniendo en cuenta que el factor anatómico en las malformaciones uterinas impide el normal desarrollo y evolución del embarazo; se recomienda su correción quirúrgica endoscópica antes de toda tentativa de reproducción asistida y en general antes de una gestación natural.

PALABRAS CLAVE: Malformaciones Uterinas. Histeroscopia Operatoria. Fertilización In-Vitro.

\section{ABSTRACT}

To determine the surgical endoscopic treatment effectiveness in patients with uterine anomalies who underwent in vitro fertilization, mesured as full term pregnancy rate. A sixteen female patients with uterine anomalies cohort were studied ( $\mathrm{n}=16$ ) in a seven year period from 1990 to 1997; nine of them underwent surgical treatment $(n=9)$ and seven women were not treated $(n=7)$. In all patients one or many in vitro fertilization cycles with identic protocols were realized.

The pregnancy rate was $44,4 \%$ for the surgical treated group versus $42,8 \%$ for the non treated group, without significative statistic difference ( $p>0,005$ ). The full term pregnancy rate was $44,4 \%$ for the treated group versus $14,2 \%$ for the other.

We conclude that uterine septum endoscopic surgical treatment improves the rates of normally developed and full term pregnancies; this is probably a result of a more capable and adequate uterine cavity. We recommend the surgica endoscopic correction of uterine septum before planning a pregnancy to decrease pregnancy losses and improve full term pregnancy rates.

KEY WORDS: Uterine Malformations. Surgical Hysteroscopy.

Ginecólogo. Especialista en Medicina Reproductiva. Prof. Servicio de Medicina Reproductiva, dpto. Gineco-obstetricia. UIS. Bucaramanga. Colombia.

Jefe servicio de medicina reproductiva. Dpto Gineco-obstetricia. UIS.

Gineco-obstetra. Epidemiólogo clínico. Dpto. Gineco-obstetricia. UIS.

Prof. Servicio ultrasonografía. Dpto. Gineco-obstetricia. UIS.

Médico Cirujano. UIS.

Jefe de servicio Ginecología B. Hôpital Universitaire de la Conception. Marseille. France.

Jefe de servicio Ginecología C. Hôpital Universitaire de la Conception. Marseille. France.

\section{Introducción}

La organogénesis de las vías genitales se desarrolla de manera concomitante con la de las vías urinarias entre la $3^{\text {a }}$ y $17^{\text {a }}$ semanas de la vida fetal. Inicialmente los embriones poseen dos sistemas pares de conductos genitales: los canales de Wolff que constituyen la vía excretora del mesonefros o riñón primitivo y los canales de Muller o paramesonefricos que se fusionan y terminan en el canal útero-'vaginal en la diferenciación femenina.

Esta embriogénesis se divide en tres fases: 
La fase genito-urinaria: de la sexta a la novena semanas de vida embrionaria, corresponde a la formación de las vías urinarias y la formación de los canales de Muller.

La fase de plegamiento: de la décima a la doceava semana de vida embrionaria, corresponde al plegamiento de las porciones cervico-vaginal de los canales de Muller, seguida del plegamiento de las porciones uterinas.

La fase de resorción: de la $13^{\mathrm{a}}$ a la $17^{\mathrm{a}}$ semanas de la vida embrionaria, corresponde a la resorción del tabique que separa los dos canales de Muller.

El tipo de malformación uterina observado es así directamente correlacionado con la época de aparición de la anomalía en la embriogénesis, Así mismo, si el evento se produce antes de la novena semana de vida embrionaria, la malformación uterina puede estar asociada a una malformación urinaria.

\section{Clasificaciones de las malformaciones uterinas}

Clasificación de Musset: Musset y Belaich en 1964 (1) establecieron una clasificación en función de la época de advenimiento de la alteración durante la embriogénesis. Dividieron el trastorno en cuatro grandes familias: Las agenesias uterinas, las hemimatrices, los úteros tabicados y los úteros comunicantes..

\section{Clasificación de Buttram}

La clasificación de Buttram (2) es la clasificación más utilizada por los autores anglosajones:

Clase I: Agenesias e hipoplasias mullerianas.

Clase II: Uteros unicornes.

Clase III: Úteros bidelfos.

Tabla 1

FRECUENCIA RELATIVA DE LAS MALFORMACIONES UTERINAS SEGUN AUTORES FRANCESES

\begin{tabular}{|c|c|c|c|c|}
\hline AUTOR & N DE CASOS & $\begin{array}{c}\text { UNICORNES } \\
\text { PSEUDO- } \\
\text { UNICORNES }\end{array}$ & BICORNES & TABIQUES \\
\hline $\begin{array}{c}\text { MUSSET } \\
1964(1)\end{array}$ & 80 & 15 & 26 & 39 \\
\hline $\begin{array}{c}\text { GANIER } \\
1972(29)\end{array}$ & 100 & 3 & 65 & 32 \\
\hline $\begin{array}{c}\text { VITSE } \\
1973(30)\end{array}$ & 100 & 8 & 40 & 52 \\
\hline $\begin{array}{c}\text { ROCHET } \\
\text { 1983 (31) }\end{array}$ & 155 & 94 & 16 & 45 \\
\hline $\begin{array}{c}\text { VALDES } \\
1987(32)\end{array}$ & 33 & 2 & 13 & 18 \\
\hline LECOUTOUR & & & & \\
\hline 1986(11) & 65 & 9 & 19 & 37 \\
\hline RUDIGOZ & 135 & 22 & 41 & 72 \\
\hline $1990(20)$ & 668 & $153(22.9 \%)$ & $220(32.9 \%)$ & $295(44.1 \%)$ \\
\hline TOTAL & & & \\
\hline
\end{tabular}

Clase IV: Uteros bicornes.

Clase V: Úteros tabicados.

Clase VI: Que agrupan las anomalías ligadas al síndrome Dietil-etil-bestrol.

\section{Frecuencia}

La frecuencia real de las malformaciones uterinas permanece desconocida debido la existencia de numerosos casos asintomáticos. Los cálculos estimados según los artículos publicados en los años 70 y 80 concluyen:

Una incidencia de $1 / 200$ a $1 / 600$ en mujeres en edad de procrear o sea una frecuencia de 0,2 a $0,5 \%$ (3 - 5). Varios estudios más recientes que incluyen a las mujeres listas para esterilización tubárica o en el marco de un seguimiento sistemático se encuentran cifras mucho más elevadas: 1,9\% para Ashton (6), 4,8\% para Sorensen (7), $3 \%$ para Nasri (8); de tal manera que con las cifras dadas por la literatura, la frecuencia global puede ser evaluada en $2 \%$. El único medio para conocer la verdadera frecuencia sería el de realizar de manera sistemática histerosalpingografía a un basto grupo de mujeres en edad reproductiva lo que es totalmente irrealizable. La frecuencia de las diferentes malformaciones uterinas según los autores anglosajones y franceses, se discrimina en las tablas 1 y 2 ; resaltando que el tabique es la malformación uterina más frecuente.

\section{Diagnóstico}

Eventualmente puede ser evocado por la clínica pero el diagnóstico exacto de la malformación uterina reposará sobre la convergencia de exámenes complementarios.

Existen algunas circunstancias en que las anomalías uterinas pueden ser sospechadas clínicamente ya que estas en su mayor parte son asintomáticas: Como cuando hay historia de dismenorrea primaria, dispareunia, amenorrea o infertilidad; cuando existen antecedentes obstétricos tales como abortos a repetición, partos prematuros, presentaciones distocicas, retardo del crecimiento, muertes fetales en el útero o accidentes del alumbramiento.

Tabla 2

FRECUENCIA RELATIVA DE LAS MALFORMACIONES UTERINAS SEGUN AUTORES ANGLOSAJONES

\begin{tabular}{|c|c|c|c|c|}
\hline AUTOR & No DE CASOS & $\begin{array}{l}\text { UNICORNES Y } \\
\text { PSEUDO- } \\
\text { UNICORNES. } \\
\text { CLASE I }\end{array}$ & $\begin{array}{l}\text { BICORNES. } \\
\text { CLASE III Y IV }\end{array}$ & $\begin{array}{l}\text { TABIQUES. } \\
\text { CLASE V }\end{array}$ \\
\hline $\begin{array}{l}\text { MUSICH } \\
1978 \text { (28) }\end{array}$ & $46^{\circ}$ & 1 & 29 & 16 \\
\hline $\begin{array}{l}\text { BUTTRAM } \\
1979(2)\end{array}$ & 91 & 19 & 5 & 67 \\
\hline $\begin{array}{c}\text { MICHALAS } \\
1991 \text { (16) }\end{array}$ & 57 & 9 & 36 & 12 \\
\hline $\begin{array}{l}\text { SIMON } \\
1991 \text { (33) }\end{array}$ & 22 & - & 2 & 20 \\
\hline TOTAL & 258 & $31(12 \%)$ & $101(39 \%)$ & $126(49 \%)$ \\
\hline
\end{tabular}




\section{Exámenes complementarios}

El objetivo es distinguir tres grandes categorías de malformaciones uterinas.

Úteros unicornes o pseudounicornes.

Úteros bicornes.

Úteros tabicados.

Para hacerlo disponemos de cuatro exámenes paraclínicos que se complementan entre sí.

Ecografía pélvica: Determina los contornos externos del útero de una manera precisa. Cuando se realiza en la segunda parte del ciclo, en el primer trimestre del embarazo o en el postparto inmediato, permite el contraste ofrecido por el endometrio hipoecogénico para analizar los contornos internos de la cavidad uterina, con la vejiga completamente llena en algunas ocasiones se puede determinar la existencia de úteros bicornes; la ecografia además permite también evaluar el estado de los riñones.

Histerosalpingografía: Esta permite la visualización de una o de las cavidades uterinas. Si las dos Hemicavidades son muy divergentes con un ángulo de separación superior a los cien grados y si son poco divergentes con un ángulo inferior a noventa grados podemos, concluir (aunque no siempre), que en el primer caso se trata de úteros bicornes y en el segundo de úteros tabicados; este examen no es suficiente por sí solo para clasificar el tipo de malformación debido a que no visualiza los contornos externos del útero.

Histeroscopia: Explora de una manera muy precisa la cavidad uterina pero por las mismas razones que la histerosalpingografía este examen no es suficiente para diagnosticar el tipo exacto de la malformación. La principal ventaja de la vía endoscópica es que permite realizar al mismo tiempo la correción quirúrgica de la anomalía.

Laparoscopia: Ayuda a diagnosticar el tipo de malformación ya que determina de una manera clara los contornos del útero y ayuda al diagnóstico de eventuales lesiones asociadas.

\section{Efectos sobre la fertilidad}

Es clásico admitir que las mujeres portadoras de una malformación uterina no tienen mayor dificultad de concebir que las demás, pero tienen más riesgos para el mantenimiento del embarazo y su buen desarrollo.

Consideremos ahora la incidencia de problemas de infertilidad encontrados en pacientes que presentan malformaciones uterinas; las cifras observadas por diferentes autores son muy dispersas (tabla 3). Las variaciones entre las cifras observadas podrían estar ligadas a sesgos en el reclutamiento de las pacientes para ciertos de esos equipos; de tal manera que el porcentaje de mujeres infértiles dentro del grupo de pacientes con malformaciones uterinas varía entre 6,1 y $41,4 \%$. En dos estudios encontrados $(9,11)$ se observa que en pacientes con M.U. la infertiidad es primaria en un $15-30 \%$ de las veces y es secundaria en 6 a $8 \%$ de las veces.

Etiología de la infertilidad en pacientes portadoras de una malformación uterina: Al respecto se han encontrado tres estudios (tabla 4). Entre las causas mencionadas podemos observar un predominio de la etiología tubárica y de la endometriosis y solamente siete casos de causa tubárica aislada. La proporción de pacientes que presenta una causa hormonal permanece bastante importante: $20.4 \%$. De otro lado, el $20 \%$ de los casos presenta una infertilidad inexplicada, grupo en el cual la malformación uterina podría estar implicada como causa de infertilidad

\section{Efectos obstétricos de las malformaciones uterinas}

Hay diversos factores que pueden explicar los efectos obstétricos desfavorables en pacientes que presentaron una malformación uterina.

1. Factores primarios.

a. Defecto de la ampliación (cavidad uterina inadecuada).

b. Defecto de la vascularización.

c. Anomalías de la contractilidad del miometrio.

d. Anomalías asociadas: cuerno uterino rudimentario, etc.

e. Anomalías renales.

f. Comunicación entre hemicavidades con fragilización del tejido endometrial.

2. Anomalías secundarias: Estas pueden ser debidas a accidentes obstétricos o a sus tratamientos.

a. Incompetencia cervico istmica secundaria a curetajes iterativos.

b. Sinequias uterinas.

c. Lesiones tubáricas.

Los efectos sobre el primer trimestre del embarazo son:

Abortos espontáneos: Su frecuencia es mucho más elevada que en la población general entre el 30 y $50 \%$ con una desviación estándar que varía entre el 19 y $67 \%$ según las series. (tabla 5). El carácter fibroso y poco vascularizado del tabique explica sus consecuencias obstétricas.

Embarazos extrauterinos: Su frecuencia entre los pacientes con malformaciones uterinas es muy parecida

Tabla 3

PROPORCION DE MUJERES INFERTILES QUE PRESENTAN UNA MALFORMACION UTERINA

\begin{tabular}{|c|c|c|c|}
\hline AUTORES & $\begin{array}{l}\text { N` DE PACIENTES } \\
\text { CON } \\
\text { MALFORMACIONE } \\
\text { S UTERINAS. }\end{array}$ & $\begin{array}{l}N^{\circ} \text { DE PACIENTES } \\
\text { INFERTILES }\end{array}$ & $\%$ \\
\hline $\begin{array}{l}\text { MUSICH } \\
1978(28)\end{array}$ & 49 & 3 & $6.1 \%$ \\
\hline $\begin{array}{c}\text { HEINONEN } \\
1983(5)\end{array}$ & 208 & 19 & $9,1 \%$ \\
\hline $\begin{array}{c}\text { MICHALAS } \\
\text { 1992(16) }\end{array}$ & 62 & 5 & $8 \%$ \\
\hline $\begin{array}{c}\text { LECOUTOUR } \\
\text { 1987(11) }\end{array}$ & 65 & 14 & $21,5 \%$ \\
\hline $\begin{array}{l}\text { FEDELE } \\
1987(19)\end{array}$ & 19 & 6 & $31.6 \%$ \\
\hline $\begin{array}{c}\text { MOUTOS } \\
1992(9)\end{array}$ & 58 & 24 & $41,4 \%$ \\
\hline
\end{tabular}


Tabla 4

ETIOLOGIA DE LA INFERTILIDAD EN PACIENTES CON M.U.

\begin{tabular}{|c|c|c|c|c|c|c|c|}
\hline AUTORES & UTERINA & HORMONAL & TUBARICA & $\begin{array}{c}\text { ENDOME- } \\
\text { TRIOSIS }\end{array}$ & MASCULINA & INEXPLICADA & $\begin{array}{l}\text { TOTAL } \\
\text { CASOS }\end{array}$ \\
\hline HEINONEN (5) & 2 & 8 & 1 & 2 & 1 & 5 & 19 \\
\hline $\begin{array}{c}\text { FEDELE } \\
\text { (19) }\end{array}$ & - & - & 1 & 3 & - & 2 & 6 \\
\hline $\begin{array}{c}\text { MOUTOS } \\
\text { (9) }\end{array}$ & - & 2 & 5 & 11 & 2 & 4 & 24 \\
\hline TOTAL & $\begin{array}{c}2 \\
(4 \%)\end{array}$ & $\begin{array}{c}10 \\
(20,4 \%)\end{array}$ & $\begin{array}{c}7 \\
(14,3 \%)\end{array}$ & $\begin{array}{c}16 \\
(32,6 \%)\end{array}$ & $\begin{array}{c}3 \\
(6,1 \%)\end{array}$ & $\begin{array}{c}11 \\
(22,4 \%)\end{array}$ & 49 \\
\hline
\end{tabular}

Tabla 5

FRECUENCIA DE ABORTOS DEL PRIMER TRIMESTRE SEGUN NUMERO DE EMBARAZOS NORMALES POR TIPO DE MALFORMACION UTERINA

\begin{tabular}{|c|c|c|c|c|c|c|}
\hline \multirow[t]{2}{*}{ AUTOR } & \multicolumn{2}{|c|}{$\begin{array}{c}\text { UNICORNE Y } \\
\text { PSEUDOUNICORNE }\end{array}$} & \multicolumn{2}{|c|}{ BICORNE } & \multicolumn{2}{|c|}{ TABICADO } \\
\hline & EMBARAZOS & ABORTOS & EMBARAZOS & ABORTOS & EMBARAZOS & ABORTOS \\
\hline ANDREWS (18) & 13 & 7 & - & - & - & - \\
\hline HEINONE ( 5 ) & 15 & 7 & 169 & 48 & 81 & 21 \\
\hline BUTRAM (2) & 119 & 39 & 43 & 163 & 208 & 139 \\
\hline FEDELE (19) & 29 & 15 & - & - & - & - \\
\hline LUDMIR (10) & 5 & 1 & 71 & 21 & 35 & 5 \\
\hline RUDIGOZ (20) & 15 & 13 & 79 & 13 & 162 & 72 \\
\hline MICHALAS (16) & 21 & 5 & 71 & 22 & 35 & 19 \\
\hline MOUTOS (9) & 40 & 1 & 18 & 6 & - & - \\
\hline TOTAL & 297 & 98 & 845 & 273 & 521 & 256 \\
\hline$\%$ & \multicolumn{2}{|c|}{$33 \%$} & \multicolumn{2}{|c|}{$32,3 \%$} & \multicolumn{2}{|c|}{$49,1 \%$} \\
\hline
\end{tabular}

a la observada en la población general con un riesgo un poco más elevado en relación con el útero pseudounicorne, porque el embarazo puede ubicarse sobre el cuerno rudimentario comportándose como un embarazo ectópico con muchas complicaciones.

En el segundo trimestre del embarazo la tasa de abortos tardíos es mucho más elevada comparativamente con la de la población general: $3 \%$ al $15 \%(9-13)$. Los abortos tardíos pueden ser debidos a una cavidad uterina inadecuada o a una incompetencia cervico-itsmica como consecuencia de curetajes repetidos; Heinonen (5) reporta un neto aumento de la tasa de sobrevivencia fetal después del cerclaje cervical, procedimiento que él recomienda en caso de úteros bicornes. 
Lecoutour (11) compara la tasa del $12,5 \%$ de abortos espontáneos después del cerclaje cervical Vs $50,4 \%$ de abortos sin cerclaje.

En el tercer trimestre del embarazo las malformaciones uterinas tienen repercusiones obstétricas que resultan de la disarmonía entre la capacidad uterina y el volumen fetal. Se ha notado una mayor frecuencia de toxemias gravídicas (11\%), retardo del crecimiento intrauterino, y desprendimiento prematuro de la placenta; fenómenos imputables todos a la anomalía de la perfusion útero-placentaria. Presentaciones distócicas con una frecuencia cercana al $30 \%$ en la que la mayoría son presentaciones caudales $(25 \%)$; explicando de la misma manera la mayor frecuencia de cesáreas en caso de malformaciones uterinas.

La prematurez constituye el riesgo principal de estos embarazos; las tasas de prematurez en efecto son mucho más elevadas con relación a la población general aproximadamente $25 \%$ Vs 5\% - 6\% (9-11, 14-16).

En el curso del parto los autores reportan una mayor frecuencia de distocia dinámica, numerosas situaciones patológicas y de presentaciones distócicas que explican el uso mas frecuente de cesáreas; cualquiera que sea el tipo de malformación; las hemorragias del alumbramiento son mas frecuentes explicadas por la anomalía en la contractilidad del miometrio.

\section{Posibilidades terapéuticas}

Solo el tratamiento quirúrgico, donde el objetivo es la reconstrucción de una cavidad uterina anatómica y funcionalmente normal, representa una real posibilidad terapéutica; este tratamiento es esencialmente mas efectivo en los úteros tabicados.

Existen diferentes procedimientos quirúrgicos:

Las técnicas quirúrgicas clásicas que necesitan frecuentemente una laparotomía y una histerotomía; y las técnicas quirúrgicas endoscópicas de aparición mas reciente en donde los excelentes resultados y su inocuidad hacen de éste el tratamiento de primera intención en el momento actual.

Técnicas quirúrgicas clásicas:

a. Cirugía de Strassmann: Descubrió la técnica para la reunificación de dos hemimatrices con cuello único inicialmente en 1907 y posteriormente con extensión a úteros tabicados (1). Los resultados informados llevaron rápidamente a otros autores a interesarse por este tipo de cirugía. En Francia Bret y Pálmer descubrieron otra técnica para úteros tabicados con una incisión anteroposterior del cuerpo uterino y un incisión del tabique (no excisión) con el fin de obtener una cavidad uterina lo mas grande posible.

Técnicas endoscópicas:

Las técnicas histeroscópicas propuestas inicialmente por Edstrom (17) desde 1974 estuvieron inicialmente frenadas debido a la mala calidad de la óptica, y la falta de accesorios eficaces. Posteriormente con el advenimiento de la luz fría y el desarrollo de los endoreceptores se entró a una nueva era; la histeroscopia operatoria; esta es realizada en la primera parte del ciclo espontáneo o mejor después de una preparación del endometrio con progestágenos, Danazol o análogos de la GnRH. La cirugía puede realizarse bajo anestesia general o local; para seguir continuamente esta maniobra operatoria intrauterina la mayoría de los autores utilizan ecoguidaje peri-operatorio con el objetivo de respetar un muro de seguridad fundico; Se aconseja también no empujar el receptor muy lejos ya que se podría fragilizar el útero o podría dejar un fondo de útero demasiado arqueado.

Se trata de una intervención totalmente exsangüe ya que el tabique es poco vascularizado. Existen varias técnicas descritas. La técnica mas frecuentemente utilizada es la sección eléctrica del tabique con el resectoscopio.

Existen otras posibilidades endoscópicas que permiten obtener resultados equivalentes:

a. La histeroscopia clásica con tijeras rígidas.

b. La sección con Nd Yag Láser (Neodymiun Yttrium Aluminium Garnet) sistema que se puede adaptar al fibroscopio, necesitándose una dilatación de $6 \mathrm{~mm}$ solamente para su introducción cualquiera que sea la técnica utilizada (21).

El resultado anatómico será juzgado por un control histeroscópico o histerográfico mas o menos tres meses después.

Histerosplastia por vía endoscópica: Revisión de la literatura: En relación con el resultado funcional todos los autores están de acuerdo en decir que estos son excelentes, (tabla 6). De la tabla se puede observar que las tasas de mujeres embarazadas después del tratamiento endoscópico para malformaciones uterinas varían entre $83 \%$ y $93 \%$, el número de abortos espontáneos varía entre $7,6 \%$ y $31 \%$, las tasas de embarazo evolutivo o sea de embarazos que evolucionan mas del primer trimestre varían entre $77,7 \%$ a $93,7 \%$.

\section{Objetivo del presente estudio}

Determinar la efectividad del tratamiento quirúrgico endoscopico en pacientes con malformaciones uterinas sometidas a FIV. Medida en tasas de embarazo que llegan al término.

Tabla 6

RESULTADOS DE LA HISTEROSPLASTIA ENDOSCOPICA

\begin{tabular}{|c|c|c|}
\hline \multirow{2}{*}{ AUTORES } & $\begin{array}{c}\mathbf{N}^{\circ} \text { MUJERES } \\
\text { EMBARAZADAS/N DE } \\
\text { MUJERES TRATADAS }\end{array}$ & ABORTOS ESPONTANEOS \\
\hline DE CHERNEY (23) & $67 / 72(93 \%)$ & $8(11.9 \%)$ \\
\hline FAYEZ (24) & $16 / 19(84,2 \%)$ & $2(12.5 \%)$ \\
\hline VALLE (26) & $10 / 12(83 \%)$ & $2(20 \%)$ \\
\hline MARCH (25) & $57 / 66(86.4 \%)$ & $8(14 \%)$ \\
\hline DALY (22) & $47 / 51(92 \%)$ & $15(31,9 \%)$ \\
\hline CHOE (21) & $13 / 14(92,8 \%)$ & $1(7.6 \%)$ \\
\hline TOTAL & $210 / 2.34(89.7 \%)$ & $36(17,1 \%)$ \\
\hline
\end{tabular}


Diseño: Se trata de un estudio retrospectivo en un período de siete años de 1990 a 1997.

Materiales y métodos: Estudio colaborativo con el Centre des Procreations Medicallement Assistèes Hopital Universitaire de la Conception. Marseille-Francia. Y el Servicio de Endocrinología Reproductiva Dpto de Gineco-obstetricia. Universidad Industrial de Santander. Bucaramanga - Colombia.

Se trabajó sobre un grupo de pacientes que presentan una malformación uterina y que se beneficiaron de uno o varios intentos de fertilización in-vitro. Se encontraron 38 casos de malformaciones uterinas, diagnosticados con por lo menos dos de los exámenes paraclínicos de los tres clásicos: histeroscopia, histerosalpingografía y laparoscopia. De los 38 casos de malformaciones uterinas se encontraron 16 pacientes con tabiques uterinos como única malformación y 22 pacientes con otras malformaciones: úteros pseudounicornes, unicornes y bicornes.

Para efecto del presente estudio se tomaron los 16 pacientes con tabiques uterinos; por ser esta la malformación uterina mas observada y la que con mayor frecuencia es tratada quirúrgicamente. Para responder a la pregunta de si el tratamiento quirúrgico endoscópico mejora o no el resultado funcional (tasa de embarazos a término después de F.I.V.) se estratificaron en dos grupos: Uno que recibió tratamiento quirúrgico endoscopico y el otro sin tratamiento quirúrgico; todos los pacientes fueron sometidos posteriormente a idénticos protocolos de F.I.V; para finalmente comparar los resultados entre ambos grupos.

\section{Resultados}

Se utilizaron los test estadísticos de Chi cuadrado, Chi cuadrado con corrección de Yates y test de Fisher, teniendo en cuenta el tamaño de la muestra. Un total de 38 casos presentaron malformaciones uterinas de los cuales 16 presentaban tabiques intrauterinos y 22 otras malformaciones.
De los 16 pacientes con tabiques uterinos, 9 fueron tratados con cirugía y los 7 restantes se dejaron sin tratamiento quirúrgico.

Posteriormente a todas las pacientes se les realizó por 1o menos un intento de FIV, La edad media de las pacientes fue de 33,4 años (corresponde a la edad media de todas la mujeres tratadas con FIV).

Malformaciones uterinas encontradas: Se encontraron 16 pacientes con tabique intrauterino; 9 de ellos fueron tratados quirúrgicamente por medios endoscópicos antes de intentar fertilización invitro.

El control histerosalpingográfico realizado posteriormente fue juzgado satisfactorio.

Los otros 7 pacientes no fueron manejados quirúrgicamente por diferentes razones: el tabique era muy pequeño $(n=1)$, diagnóstico tardío durante el curso de la FIV $(n=2)$, tabique amplio pero con trastornos masculinos de la fertilidad $(n=1)$, tabiques parciales en quienes no quedo claro su exclusión de la cirugía $(n=2)$.

Los 22 casos de otras malformaciones no fueron tenidas en cuenta en el presente estudio; ya que la gran mayoría no había recibido ningún tipo de tratamiento quirúrgico y el tipo de anomalía resultaba muy heterogéneo.

En el grupo de pacientes con tabiques tratados quirúrgicamente se encontró $66,7 \%$ de casos de infertilidad primaria, vs. $71.4 \%$ en el grupo de tabiques no tratados. (Tabla 7 ).

Las indicaciones de fertilización in-vitro (tabla 7). Estuvieron dominadas por las patologías tubáricas exclusivas o asociadas, seguidas de factor masculino.

Todos los protocolos de estimulación ovárica realizados en estos grupos de pacientes fueron idénticos; protocolos largos con análogos de GnRH y FSH purificada. El seguimiento se realizó con estradiolemia y ecografía trans-vaginal. Los resultados de fertilización in-vitro son mostrados de manera global (tabla 8); allí podemos observar que los grupos en estudio han respondido de manera similar al protocolo de estimulación mencionado. De las 9 pacientes del grupo de tabiques tratados quirúrgicamente se obtienen cuatro embarazos, tasa de

Tabla 7

DESCRIPCION DE LA POBLACION ESTUUDIADA. (INDICACION DE F.I.V.)

\begin{tabular}{|c|c|c|c|c|c|c|}
\hline MALFORMACION & $\begin{array}{c}\mathbf{N}^{\circ} \text { DE } \\
\text { PACIENTES }\end{array}$ & $\begin{array}{c}\text { No DE } \\
\text { INFERTILIDAD } \\
\text { PRIMARIA }\end{array}$ & $\begin{array}{c}\text { EXCLUSIVA } \\
\text { TUBARICA }\end{array}$ & $\begin{array}{c}\text { TUBARICA } \\
\text { ASOCIADA A } \\
\text { OTRA }\end{array}$ & MASCULINA & INEXPLICADA \\
\hline $\begin{array}{c}\text { TABIQUES } \\
\text { TRATADOS }\end{array}$ & 9 & $6(66,7 \%)$ & 4 & 2 & 2 & 1 \\
\hline $\begin{array}{c}\text { TABIQUES NO } \\
\text { TRATADOS }\end{array}$ & 7 & $5(71.4 \%)$ & 1 & 1 & 3 & 2 \\
\hline TOTAL & 16 & $11(69 \%)$ & 5 & 3 & 5 & 3 \\
\hline
\end{tabular}


Tabla 8

RESULTADOS DE NUESTRO ESTUDIO EN PACIENTES CON M.U. CON Y SIN TRATAMIENTO QUIRURGICO, SOMETIDAS A F.I.V-E.T.

\begin{tabular}{|c|c|c|c|}
\hline & $\begin{array}{c}\text { TABIQUES } \\
\text { TRATADOS }\end{array}$ & $\begin{array}{c}\text { TABIQUES NO } \\
\text { TRATADOS }\end{array}$ & TOTAL \\
\hline $\mathrm{N}^{\circ}$ DE PACIENTES & 9 & 7 & 16 \\
\hline $\mathrm{N}^{\circ}$ DE PUNCIONES & 23 & 26 & 49 \\
\hline $\begin{array}{c}\mathrm{N}^{\circ} \\
\text { TRANSFERENCIAS }\end{array}$ & 20 & 24 & 44 \\
\hline $\mathrm{N}^{\circ}$ DE OVOCITOS & 198 & 211 & 409 \\
\hline $\mathrm{N}^{\circ}$ DE EMBRIONES & 105 & 122 & 227 \\
\hline $\mathrm{N}^{\circ}$ DE EMBRIONES \\
TRANSFERIDOS
\end{tabular}

embarazos $(44,4 \%)$; de los cuales cuatro niños vivos en casa $(44,4 \%)$; de los 7 casos de tabiques no tratados quirúrgicamente se obtienen tres embarazos, tasa de embarazo $(42,8 \%)$; de los cuales un solo bebe llega a término vivo en casa, $(14,2 \%)$; los otros dos presentaron aborto de primer trimestre; observándose que la tasa de embarazo es similar en los grupos $(44,4 \%$ Vs 42,8$)$, pero la tasa de embarazos evolutivos normales que llegan a término es significativamente mayor en el grupo de pacientes tratados quirúrgicamente $(44,4 \%)$ en comparación con el grupo no tratado (14,2\%). Evidenciando que el factor anatómico (estrechez, obstáculo, etc.), es definitivo en la evolución de los embarazos asociados a M.U.

\section{Discusión}

Si la fecundidad de las mujeres que presentan una malformación uterina parece sujeto de controversia; el pronóstico de la fecundación invitro en estas pacientes portadoras de una anomalía es aún menos bien establecido. Es conocido que la fisiopatología de los trastornos de reproducción en pacientes con malformaciones uterinas incluye:

Un defecto de la vascularización del tabique que impediría una buena implantación y un buen desarrollo del huevo.

Una estrechez extrema de la cavidad uterina que dificultaría un buen desarrollo del embarazo.

Una incompetencia cervico-itsmica que obstacularizaría el mantenimiento de una correcta gestación.

Una mayor decidualización endometrial en el sitio del tabique.
Estos hallazgos fisiopatológicos hacen pertinente el inicio de un tratamiento quirúrgico de la malformación.

La elección del tipo de tratamiento quirúrgico a efectuar a pacientes con tabique uterinos es muy sencilla ya que son numerosas las ventajas que tiene el tratamiento quirúrgico endoscópico sobre el quirúrgico clásico, a saber:

- Ausencia de laparotomía, por lo tanto reducción de la morbi-mortalidad y de la estancia hospitalaria.

- Postoperatorio muy sencillo.

- Reducción de los riesgos de las adherencias postquirúrgicas que es un factor eventual de infertilidad posterior.

- Ausencia de cicatriz uterina.

- Acortamiento en el tiempo para la decisión de un embarazo posterior.

- Por otra parte los excelentes resultados registrados con la técnica endoscópica como su facilidad, la inocuidad del tratamiento, el costo reducido y el retorno rápido a su actividad diaria normal y la disminución significativa de la morbi-mortalidad vuelve caduco las prácticas de metroplastia clásica por laparatomía

En relación con el resultado funcional todo el grupo de autores estudiados (22-27), están de acuerdo para decir que son excelentes. De tal manera que las tasas de embarazos en pacientes tratadas quirúrgicamente varían de $72 \%$ a $93 \%$. Por nuestra parte podemos decir que si relacionamos los procedimientos de procreación asistida (F.I.V-E.T.) con las M.U. (tabiques uterinos) en pacientes sin tratamiento quirúrgico y con tratamiento quirúrgico, observamos que las tasas de embarazo evolutivo que llegan a término, son mayores en el grupo de pacientes que han tenido tratamiento quirúrgico endoscópico; evidenciando que la adecuación de la cavidad uterina es factor decisivo en el pronóstico de la evolución del embarazo en pacientes con malformación uterina.

\section{Conclusiones}

Constamos que los pacientes que presentan un tabique uterino tratado endoscópicamente parece tener un pronóstico diferente al de los pacientes que no son tratados. Los resultados observados nos conducen a concluir que existe una indicación para el tratamiento quirúrgico endoscópico de los tabiques uterinos antes de planear un intento de fertilización in-vitro, ya que este mejora las tasas de embarazo evolutivo normal que llegan a término; al parecer por una mejoría en la capacidad y adecuación de la cavidad uterina.

Así mismo, teniendo en cuenta que el factor anatómico en las malformaciones uterinas impide el desarrollo y evolución normal del embarazo; recomendamos su corrección quirúrgica endoscópica antes de toda tentativa de reproducción asistida y en general antes del inicio de una gestación natural.

\section{Agradecimientos}

Al Pr. Roger Erny, Pr. Leon Boubli, Pr. Bernard Blanc, por todas sus enseñanzas.

Al Dr. Jean-louis Amselle, por todo su apoyo y confianza en $\mathrm{mi}$ : 


\section{BIBLIOGRAFIA}

1. Musset R., Belaich J. Nécessité D'Une classification globale des malformations uterines. XXII émes assises francaises de Gynécologie. Masson De. Paris. 1964.

2. Buttram V. Mullerian anomalies and their Management Fertil Steril 1983; 40(2): 159-163.

3. Green LK., Harris RE. Uterine anomalies: Frequency of diagnosis and associated obstetric complication. Obstet Gynecol. 1976; 47(4): 427-429.

4. Hannoun A., Khalil A., Karam K. Uterine Unification procedures post-operative obstetrical outcome. Int J Gynecol Obstet 1989; 30: 161-164.

5. Heinonen PK., Saarikosky S., Pystinen P. Reproductive performance of women with uterine anomalies. An evaluation of 182 cases. Acta Obstet Gynecol Scand 1982; 61: 157-162.

6. Ashton D., Amin HK., Richart RM., Neuwirth RS. The incidence of asymptomatic uterine anomalies in women undergoing transcervical tubal sterilization. Obstet Gynecol 1988; 72: 28-30.

7. Sorensen S. Estimated prevalence of mullerian anomalies. Acta Obstet Gynecol Scand 1988; 67: 441-445.

8. Nasrí M., Setchell M., Chard T. TRansvaginal ultrasound for diagnosis of uterine malformations. Brit J Obstet Gynecol 1990; 97 : 1043-1045.

9. Moutos DM, Damewood MD., Schalaff WD., Rock JA. A Comparison of the reproductive outcome between women with a unicornuate uterus and women with a didelphic uterus. Fertil Steril 1992; 58(1): 88-93

10. Ludmir J., Samuels P., Brooks S., Mennutí MT. Pregnancy outcome of patients with incorrected uterine anomalies managed in a high-risk obstetric setting. Obstet Gynecol 1990; 75: 906-910.

11. LecoutourX., Bourgeot P., Segard G., et Cols. L'avenir obstetrical des uterus malformes. Etude de 155 grosesses. Rev FR Gynecol Obstet 1986; 81: 357-362.

12. Poulin B. Le comportement obstetrical de L'utérus bicorne. A propos de 29 observations. Thèse Méd., Lyon, 1978.

13. Rudigoz., Gaucherand P., Dargent D. Le pronostic obstetrical des malformations uterines J Gynecol Obstet Biol Reprod 1989; 185-191.

14. Levy G. Malformations utérines et grossesses J Gynecol Obstet Biol Reprod 1986; 15: 530-532.

15. Maneshí M., Maneshí F., Fuca G. Reproductive impatrement of women with unicor nuate uterus. Acta Eur Fertil 1988; 81: 273-275.

16. Michalas SP. Outcome of pregnancy in women with uterine malformation: evaluation of 62 cases. Int Gynecol Obstet 1991; 35 : 215-219.
17. Edstrom KGB. Intrauterin surgical procedures during hysteroscopy. Endoscopy 1974; 6: 175-175.

18. Andrews MC., Jones HW. Impaired reproductive performance of the unicornuate uterus: intrauterine growth retardation, infertility, and recourent abortion in 5 cases. Am J Obstet Gynecol 1982; 144: 173-176.

19. Fedele L., Zamberlettí D., Vercellini P., Dorta M., Candianí GB. Reproductive perfomance of women with unicornuate uterus. Fertíl Steríl 1987; 47: 416-419.

20. Rudigoz RC., Gaucherand P. Retentissement obstetrical des malformations uterines. E.M.C. De Techniques. Obstetrique. 5061. A10, 1991.

21. Choe JK., Baggisch MS. Histeroscopic treatement of septate uterus with neodymium yag laser. Fertíl Steríl 1992; 57: 81-84.

22. Daly DC., Maier D., Soto-Albors C. Hysteroscopic metroplasty: six years experience Obstet Gynecol 1989; 73: 201-205.

23. De Cherney AH., Russell JB., Graebe RA., Polan M. Resectoscopic Management of mullerian fusion defects Fertíl Steríl 1986; 45: 726-728.

24. Fayez JA. Comparison between abdominal and hysteroscopy for metroplasty. Obstet Gynecol 1986; 68: 399-402.

25. March CM., Israel R. Hysteroscopic management of recurrent abortion caused by septate uterus. Am J Gynecol Obstet 1987; 156(4): 834842.

26. Valle RF., Scíarra JJ. Hysteroscopic treatment of the septate uterus. Obstet Gynecol 1986; 67(2): 253-257.

27. Janny L., Vyep Pouly JL., Dumont M., Nicollet B. Menezoty: cultures. Appoys. Diagnostiques et terapeutique contraset Fertil. Sex. 1993; 21(5): 392-394

28. Musich. Jr., Behrman SJ. Obstetric outcome before and after metroplasty in women with uterine anomalies.

29. Ganier G. Les malformations uterines congenitales et leurs consequences obstetricales. These medicale., Lyon 1972.

30. Vitse M., Boulanger JC., Manela A., Bouchez Y. Accouchement et malformations uterins. Rev. Fr. Gynecol-Obstet., 1973; 68: 483-491.

31. Rochet Y., Dargent D., Seffer P. Et al. Hysteroplasties pour hemiuterus et uterus cloisonnes. A propos de 45 cas. J Gynecol Obstet Biol Reprod 1985; 14: 227-237.

32. Valdes C., Malini S., Malinac LR. Ultrasound evaluation of female genital tract anomalies: a revue of 64 cases. Am. J. Obstet. Gynecol., 1984; 149: 285-292.

33. Simon C., Martinez L., Pardo F., Tortajada M., Pellicier A. Mullerian defects in women with normal reproductive outcome. Fertil-Steril. 1991; 56(6): 1192-1193. 